\title{
sciendo
}

\author{
BULGARIAN ACADEMY OF SCIENCES
}

CYBERNETICS AND INFORMATION TECHNOLOGIES • Volume 20, No 6

Special Issue on New Developments in Scalable Computing

Sofia $\bullet 2020$

Print ISSN: 1311-9702; Online ISSN: 1314-4081

DOI: $10.2478 /$ cait-2020-0074

\section{Finite-Temperature Single Molecule Vibrational Dynamics from Combined Density Functional Tight Binding Extended Lagrangian Dynamics Simulations and Time Series Analysis}

\author{
Bojana Koteska ${ }^{1}$, Anastas Mishev ${ }^{2}$, Ljupco Pejov ${ }^{2,3}$ \\ ${ }^{1}$ Faculty of Computer Science and Engineering, Ss. Cyril and Methodius University, Rugjer Boskovikj \\ 16, 1000 Skopje, North Macedonia \\ ${ }^{2}$ Institute of Chemistry, Faculty of Natural Sciences and Mathematics, Ss. Cyril and Methodius \\ University, POBox 162, 1001 Skopje, North Macedonia \\ ${ }^{3}$ Department of Chemistry, Bioscience and Environmental Engineering, Faculty of Science and \\ Technology, University of Stavanger, 4036 Stavanger, Norway \\ E-mails: bojana.koteska@finki.ukim.mk anastas.mishev@finki.ukim.mk ljupcop@pmf.ukim.mk
}

\begin{abstract}
Combining a computationally efficient and affordable molecular dynamics approach, based on atom-centered density matrix propagation scheme, with the density functional tight binding semiempirical quantum mechanics, we study the vibrational dynamics of a single molecule at series of finite temperatures, spanning quite wide range. Data generated by molecular dynamics simulations are further analyzed and processed using time series analytic methods, based on correlation functions formalism, leading to both vibrational density of states spectra and infrared absorption spectra at finite temperatures. The temperature-induced dynamics in structural intramolecular parameters is correlated to the observed changes in the spectral regions relevant to molecular detection. In particular, we consider a case when an intramolecular X-H stretching vibrational states are notably dependent on the intramolecular torsional degree of freedom, the dynamics of which is, on the other hand, strongly temperature-dependent.
\end{abstract}

Keywords: Atom-centered density matrix propagation, density functional tight binding, molecular dynamics, finite-temperature vibrational dynamics, formic acid, torsional motion.

\section{Introduction}

Molecular vibrations are of crucial importance for their detection, in both gas phase (i.e., as free molecular species), or embedded in various matrices, or condensed phases. In many cases, certain intramolecular vibrational modes may serve as 
molecular fingerprints, indicating the existence of a given molecule within the explored medium. These issues are of exceptional importance, for example, in environmental science and technology. Beyond any doubt, we can understand the environment far better if we know of what it is composed. Being able to detect individual molecular species and their clusters in realistic samples under realistic (in situ) conditions is of crucial importance in this context. Various experimental vibrational spectroscopic techniques can give us information only about the differences between energy levels of intramolecular oscillators, but they cannot tell us anything about the nature of the oscillator itself. Much of the interpretation of experimental vibrational spectroscopy data is, therefore, based on empirical data and relationships. The advent of numerous advanced computer codes for quantum mechanical modelling of molecular species, at different level of sophistication, has led to quite an improvement in theoretical support for such empirical "assignments" of signals (vibrational spectral bands) in the experimental spectrograms.

However, vibrational spectra of molecular species, computed by widelyavailable computer codes, using routine approaches, are often based on the doubleharmonic approximation (assumption of both mechanical and electrical harmonicity of the oscillator), as the advent of analytic second derivatives has undoubtedly opened a new era in molecular quantum mechanics. Aside from that, all standard ab initio molecular quantum mechanics calculations inherently refer to absolute zero $(0 \mathrm{~K})$. At the same time, experimental data are usually collected under drastically different conditions, either in gas phase, or in other condensed phases. It is therefore of crucial importance, for an in-depth understanding of the molecular vibrational spectra at finite temperature, to explicitly include the temperature in the calculations. Certain complexities and ambiguities in the interpretation of finite-temperature vibrational spectra can be resolved by adopting a systematic approach of this type.

In the present study, we focus on the investigation of vibrational dynamics of a single molecule at series of finite temperatures, ranging from close to $0 \mathrm{~K}$ up to as high as $1000 \mathrm{~K}$. To achieve this aim, we combine a computationally efficient molecular dynamics protocol, with a computationally affordable (faster up to 3-4 orders of magnitude as compared to the DFT MD methods), and yet accurate semiempirical quantum mechanics approach. We follow and correlate the temperature-induced structural dynamics changes with the spectroscopic manifestations thereof. Molecular dynamics results are analysed within the framework of time-series analytics methods.

As a particular prototype molecular system, we have chosen the simplest member of the class of compounds known as organic acids - the formic acid [1]. It is the most abundant organic acid in the Earth's atmosphere, thought to have an essential role in aerosol formation and other processes relevant to atmospheric chemistry and climate science. This simple system has also been frequently mentioned in astrochemical context [1]. Beyond all this, its behaviour reflects the basic molecular chemistry and physics of the whole class of organic acids. The present study is an extension of our previous work on this system [2]. While in [2] we have compared the performances of dynamical and anharmonic static approaches to vibrational spectroscopy of formic acid conformers in a relatively narrow 
temperature interval, in the present paper we consider a much wider temperature interval and we also consider in a somewhat more in-depth manner the timedependent changes of structural parameters thereof.

\section{Computational methodology}

\subsection{Density functional tight binding approach}

The semiempirical Density Functional Tight Binding method (DFTB [3]) was used throughout the paper for calculation of molecular electronic properties, exploration of the molecular Potential Energy Surface (PES) as well as for the molecular dynamics simulations by the extended Lagrangian approach described in the following paragraph [5]. In the current study, the DFTBA implementation of this approach in the Gaussian09 system of codes [6] has been used, which is based on analytical expressions for the matrix elements.

Full geometry optimization of formic acid starting from points on the molecular PES corresponding to the cis- and trans-conformers have been carried out with Schlegel's Gradient Optimization Algorithm [7]. Subsequently to geometry optimizations, the second-derivative matrices have been computed for the stationary points located on the PES. Absence of negative eigenvalues of the computed Hessians has been used as a confirmation that the located stationary point on the PES corresponds to a real minimum. Relaxed scans of the $\mathrm{OCOH}$ torsional potential were carried out by partial geometry optimizations, fixing the dihedral angle at series of values starting from $0^{\circ}$ to $180^{\circ}$ (with a step of $10^{\circ}$ ), while optimizing all other intramolecular parameters.

\subsection{Extended Lagrangian molecular dynamics simulations}

DFTB Molecular Dynamics (MD) simulations were carried out by the Atom-centered Density Matrix Propagation (ADMP) scheme [5], which belongs to the class of extended Lagrangian methods for MD simulations.

Six series of simulations were carried out, starting from the optimized geometries corresponding to the cis- and trans-conformers of formic acid. Appropriate amount of initial nuclear kinetic energy corresponding to the desired temperature of the system (computed from statistical mechanics principles) was injected and distributed among the atoms. ADMP MD simulations were carried out in the NVE ensemble, avoiding application of any thermostats to keep the temperature constant throughout the simulations. Such approach was implemented since the main focus of the paper is on computation of molecular vibrational dynamics at finite temperatures, within the time-correlation functions formalism. Application of thermostats could introduce severe distortions in the dynamics which would certainly affect the derived conclusions. Initial equilibration phase of the MD simulations of 2 ps was subsequently followed by production phase of 11 ps. Temperature fluctuations arising due to non-implementation of thermostats were acceptable, ranging within thermodynamically expected intervals for the apparent system's size. Cholesky decomposition scheme has been used to obtain the 
orthonormal basis set. The time step that has been used to integrate the equations of motion was set to $0.1 \mathrm{fs}$. Electron fictitious mass parameter was set to 0.1 atomic mass units (amu). The particular choices of the last two parameters were aimed to keep the adiabaticity index and the conditions for idempotency of the electronic density matrix within the literature recommended values throughout the whole trajectory.

\section{Results and discussion}

The optimized geometries of the two conformers of formic acid molecule at DFTB-A level of theory are given in Figs 1 and 2, on the other hand, shows the calculated relaxed $\mathrm{OCOH}$ torsional potential; the positions corresponding to the two minima are depicted in Fig. 1 as well.

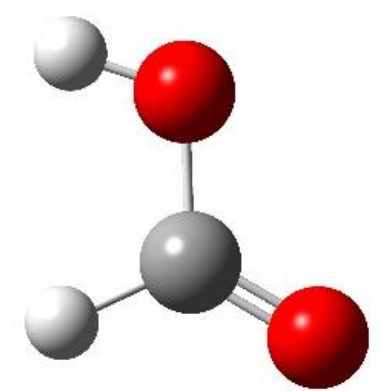

(a)

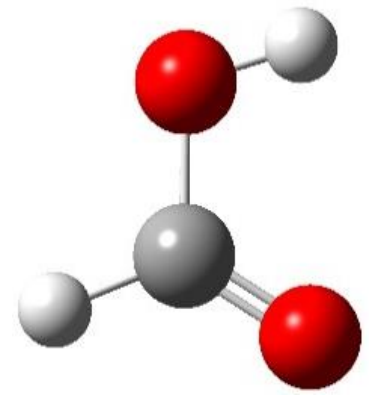

(b)

Fig. 1. The cis- (a) and trans- (b) minima located on the DFTB PES of free formic acid molecule

Both the structural and thermodynamical parameters concerning the stability of the two conformers of the free formic acid molecule agree rather well with the experimental data [8]. For example, the energy difference between the cis- and transminima computed at the DFTB level of theory is $1323.6 \mathrm{~cm}^{-1}$, i.e., $3.78 \mathrm{kcal} . \mathrm{mol}^{-1}$ without the inclusion of the Zero-Point Vibrational Energy corrections (ZPVE). Inclusion of the ZPVE corrections, within the harmonic approximation, leads to $1179.2 \mathrm{~cm}^{-1}$, i.e., $3.37 \mathrm{kcal}^{\mathrm{mol}}{ }^{-1}$, respectively. The experimental value of the last parameter is $1365 \pm 30 \mathrm{~cm}^{-1}$ [8]. The estimated barrier height for the trans $\rightarrow$ cis torsional transition from the relaxed torsional potential scan is $3430 \mathrm{~cm}^{-1}$, which compares rather well with the available experimental data for species in cryogenic $\mathrm{Ar}$ matrix of $3810 \pm 100 \mathrm{~cm}^{-1}$ [8]. Note, however, that the last value is expected to be higher than the free-molecule one due to matrix effects. All these data confirm rather good parametrization of the chosen DFTB method for the main purpose of the present study - investigation of the vibrational dynamics at finite temperatures, but still below the torsional barrier threshold. 


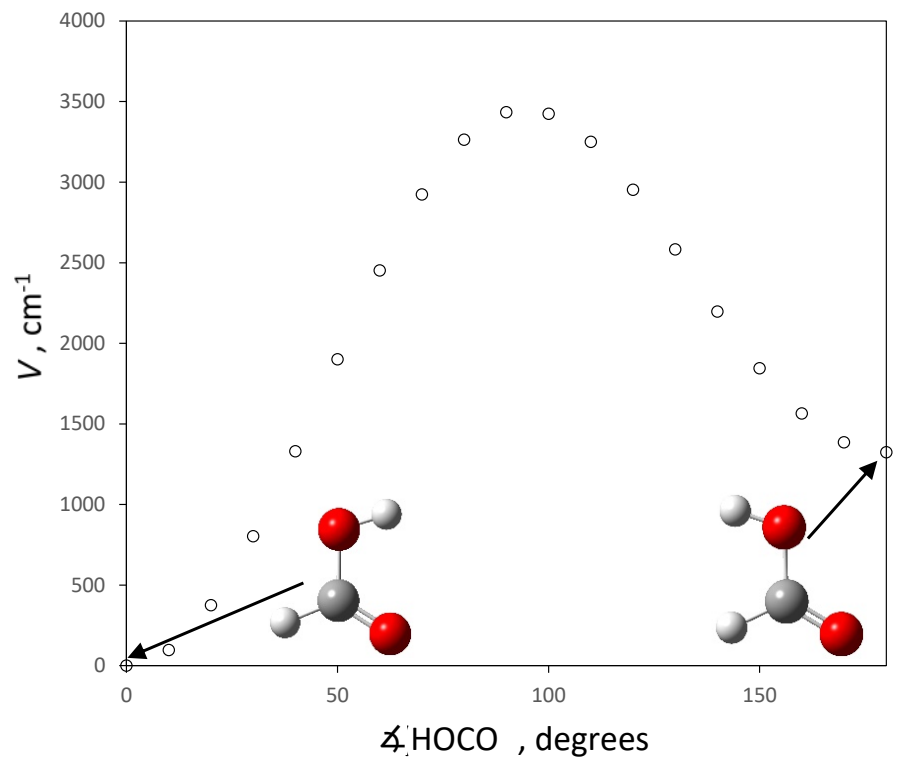

Fig. 2. The relaxed OCOH torsional potential of free formic acid molecule computed at DFTB level of theory

To illustrate the stability of ADMP trajectory, in Fig. 3 we present the timeevolution of the adiabaticity index in the first picosecond of the production part of the ADMP run for the simulation carried out at $T=5 \mathrm{~K}$ for both cis-and transconformers of the free formic acid molecule. As can be seen, the observed values are far below the proposed threshold [5], and such trend is preserved throughout the trajectory for all temperatures.

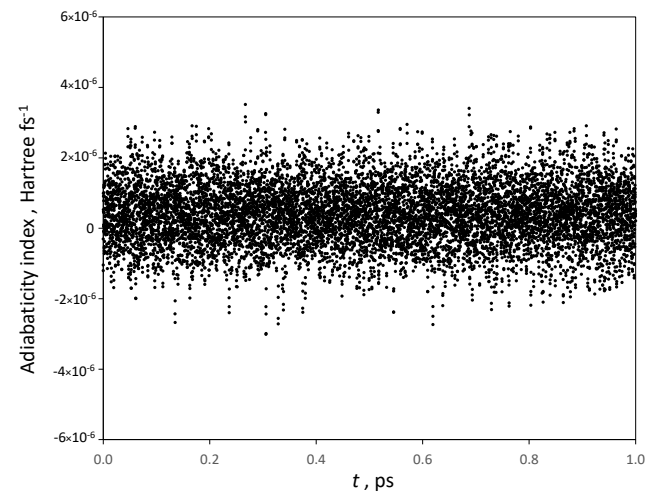

(a)

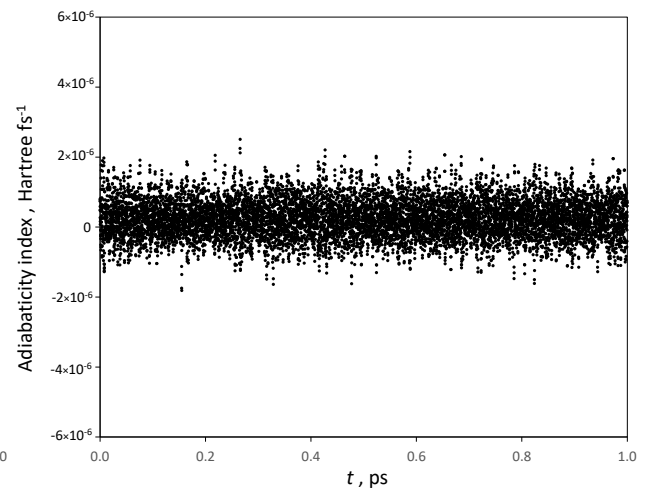

(b)

Fig. 3. Time-evolution of the adiabaticity index in the first picosecond of the production part of the ADMP run for the simulation carried out at $T=5 \mathrm{~K}$ for: cis-conformers (a), and trans-conformers (b) of free formic acid

The kinetic energy spectra, which are directly proportional to the vibrational densities of states obtained with inelastic neutron scattering, were calculated from the 
velocity-velocity autocorrelation function [9]. The last function has been calculated from the equilibrated DFTB-ADMP run as

$$
\langle\vec{v}(0) \vec{v}(t)\rangle=\lim _{T \rightarrow \infty} \sum_{i=1}^{N} \sum_{j=1}^{3} \int_{t^{\prime}=0}^{t^{\prime}=T} v_{i, j}\left(t^{\prime}\right) \cdot v_{i, j}\left(t^{\prime}+t\right) d t^{\prime}
$$

Here $v_{i, j}$ denotes the $j$-component of the velocity vector of atom $i$, while the summation is carried out up to the total number of atoms $N$. The power (intensity) spectrum, i.e., the kinetic Density Of States (DOS) or vibrational DOS spectrum is subsequently computed by the Fourier transformation:

$$
I_{v}(\omega)=\lim _{T \rightarrow \infty} \int_{t=0}^{t=T}\langle\vec{v}(0) \vec{v}(t)\rangle \cdot \exp (-i \omega t) d t
$$

In performing Fourier transformation (2), we have used the Blackman's window (apodization) function. If, instead of the velocity-velocity autocorrelation function, the dipole moment autocorrelation function is subjected to Fourier transformation in (2), upon suitable scaling with the quantum nuclear correction term, the power spectrum which is directly proportional to the experimentally measured infrared spectrum is obtained. We have performed this analysis for the purpose of the present study as well. However, since the results were analogous to those obtained directly from the velocity autocorrelation function, we will focus further only on these results. Fig. 4 shows the region of O-H stretching modes in the vibrational DOS spectra of cis- and trans- conformers of free formic acid molecule which have been computed by time-series analysis of data collected from trajectories at $5 \mathrm{~K}$.

As can be seen, in each case only a single prominent band is visible, corresponding to the $\mathrm{O}-\mathrm{H}$ vibrational stretching motion which is localized within the $\mathrm{O}-\mathrm{H}$ group in both conformers. In the case of cis-conformer, the band is centred at about $3619 \mathrm{~cm}^{-1}$, while in trans-conformer, it appears at somewhat lower wavenumber values $\left(3570 \mathrm{~cm}^{-1}\right)$. The "red-shift" of the $\mathrm{O}-\mathrm{H}$ stretching mode in the case of trans-conformer relative to the cis- is due to an intermolecular weak O-H...O contact (Fig. 1b) which imposes an environment around the O-H oscillator slightly resembling a proton-acceptor moiety in a weak hydrogen bond. In the case of cisconformer, on the other hand, the intramolecular O-H...H contact (Fig. 1a), due to the repulsive Pauli interaction steepens the $\mathrm{O}-\mathrm{H}$ stretching potential which results in a frequency "blue-shift" (relative to the trans-conformer).

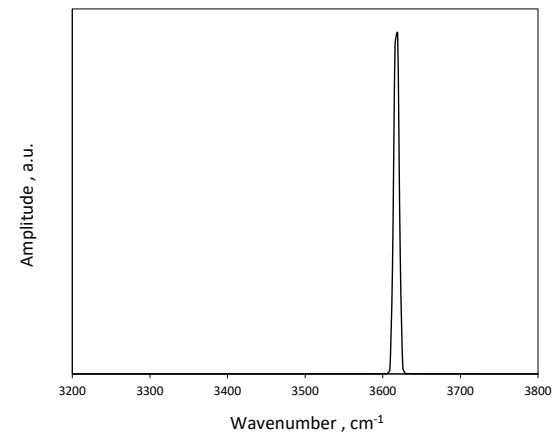

(a)

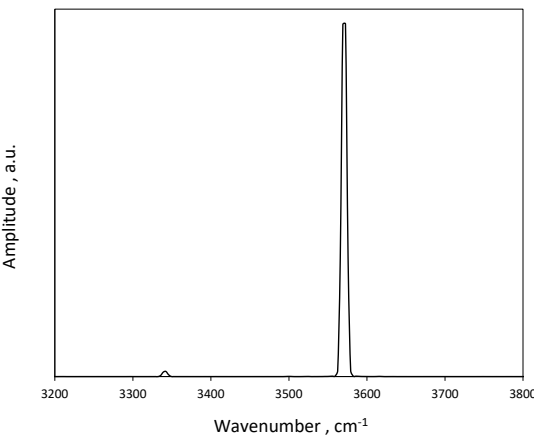

(b)

Fig. 4. The region of O-H stretching modes in the vibrational DOS spectra of: cis-conformers (a), and trans-conformers (b) of formic acid computed from time-series analysis of data collected from trajectories at $5 \mathrm{~K}$ 


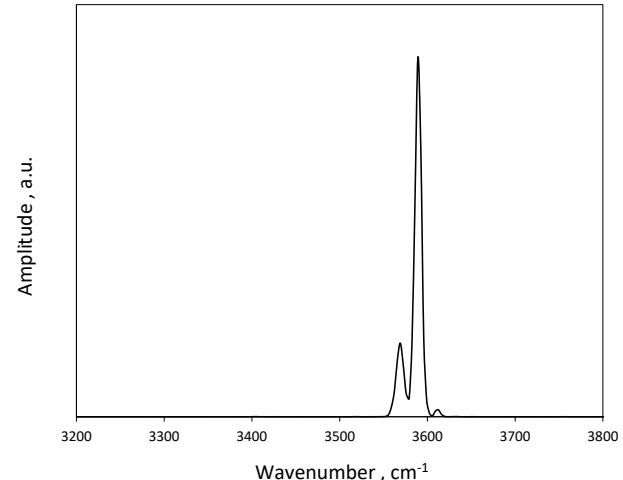

(a)

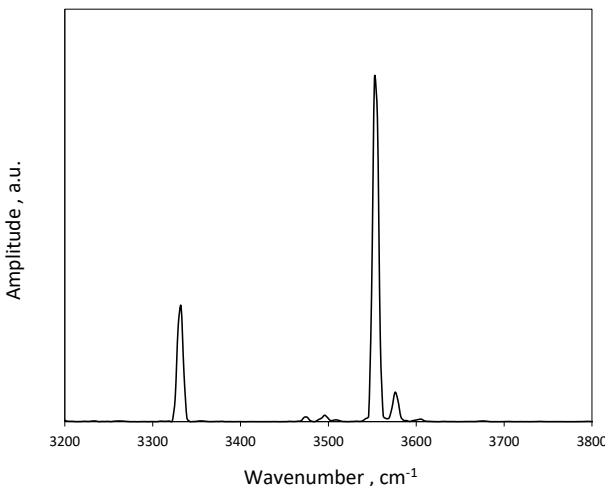

(b)

Fig. 5. The region of O-H stretching modes in the vibrational DOS spectra of cis-conformers (a), and trans-conformers (b) of formic acid computed from time-series analysis of data collected from trajectories at $300 \mathrm{~K}$

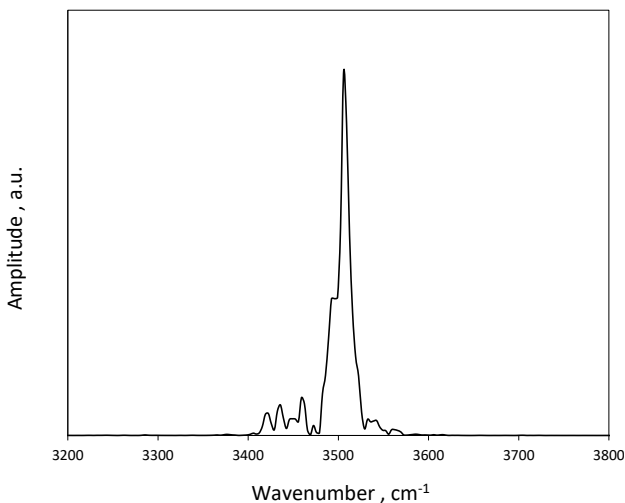

(a)

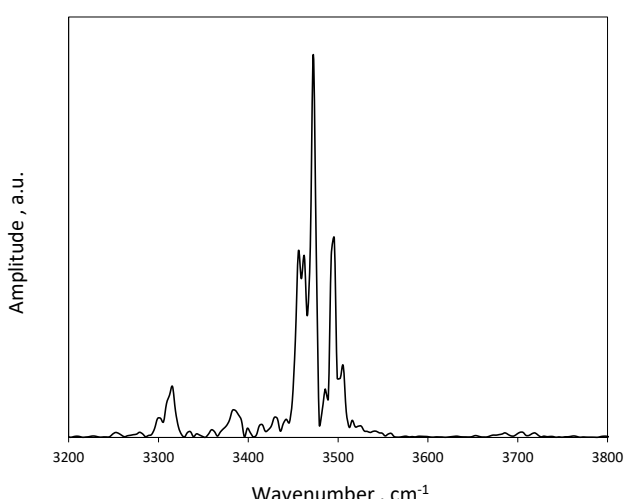

(b)

Fig. 6. The region of O-H stretching modes in the vibrational DOS spectra of cis-conformers (a), and trans-conformers (b) of formic acid computed from time-series analysis of data collected from trajectories at $1000 \mathrm{~K}$

Heating at $300 \mathrm{~K}$, introduces visible changes in the vibrational DOS spectra of both conformers (Fig. 5). As compared to the spectral appearances at $5 \mathrm{~K}$, the most prominent bands in the case of both conformers shift to lower wavenumbers. We attribute this observation to the following. The $\mathrm{O}-\mathrm{H}$ stretching oscillators are inherently characterized by huge anharmonicity. This means that the first approximation of treating the $\mathrm{O}-\mathrm{H}$ stretching mode like a harmonic oscillator fails at longer elongations (O-H bond stretches). Thus, at $300 \mathrm{~K}$, the enhanced thermal motion causes the O-H oscillator to sample more "distant" regions of its potential energy curve (with respect to the minimum), which are inherently more "anharmonic". The temperature-induced red shifts, therefore, are typical examples of a breakdown of the harmonic approximation. Appearance of additional bands in the same spectral region are induced by different orientations of the $\mathrm{O}-\mathrm{H}$ oscillator with respect to other intramolecular centers, i.e., they reflect different "local environments" experienced by this mode. Of course, one has to keep in mind that the 
present plots have been obtained from single ADMP trajectories. Averaging the data from a large number of trajectories would probably lead to appearance of wider peaks in the case of both conformers, instead of several distinct peaks. At $300 \mathrm{~K}$, in the case of cis- conformer the vibrational DOS exhibits a relatively stronger peak at lower wavenumber values and a weaker one at higher, in contrast to the case of transconformer, where only a weak band at higher frequencies is present. This is due to the fact that the potential energy well from the cis-side is shallower than from the trans-side, and therefore more "trans-like" configurations are more easily accessible from the side of the potential corresponding to the less stable structure (see Fig. 2).

At yet higher temperatures $(1000 \mathrm{~K}$, see Fig. 6), the trend is preserved. The VDOS spectra of both conformers are characterized by rather wide spectral features, appearing around $3500 \mathrm{~cm}^{-1}$, with notable substructures, which would be smeared out upon averaging the data collected from numerous trajectories.

The temperature trends discussed before in the context of VDOS spectra are in line with the geometry changes upon temperature increase. As the main focus in the present study has been put on the $\mathrm{O}-\mathrm{H}$ stretching mode, the frequency of which is heavily-dependent on the $\mathrm{OCOH}$ torsional motion, we illustrate these changes through the time-evolution of the corresponding dihedral angle in the case of both conformers. Figs 7-9 represent the time-evolution of the $\mathrm{OCOH}$ dihedral angle throughout the first picosecond of the production ADMP runs at $5 \mathrm{~K}, 300 \mathrm{~K}$ and $1000 \mathrm{~K}$, for the trajectories started from the cis- and trans-minima on the DFTB PES. Evidently, the amplitudes of oscillations of the torsional angle, as well as the average values, increase with the increase in temperature.

Table 1 summarizes the average absolute deviations of the torsional angle values computed with respect to the referent ones (corresponding to the minima on the DFTB PES).

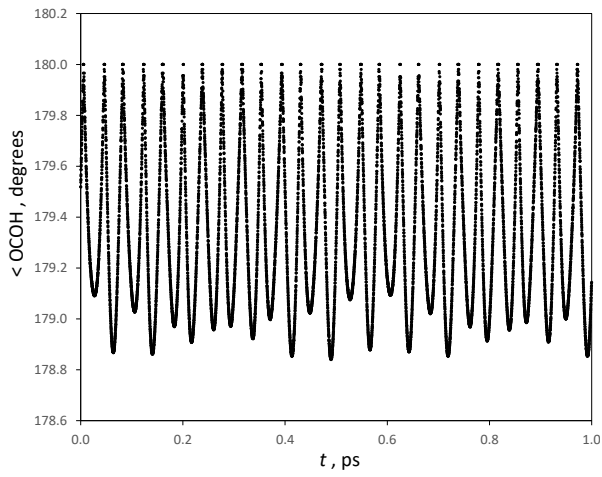

(a)

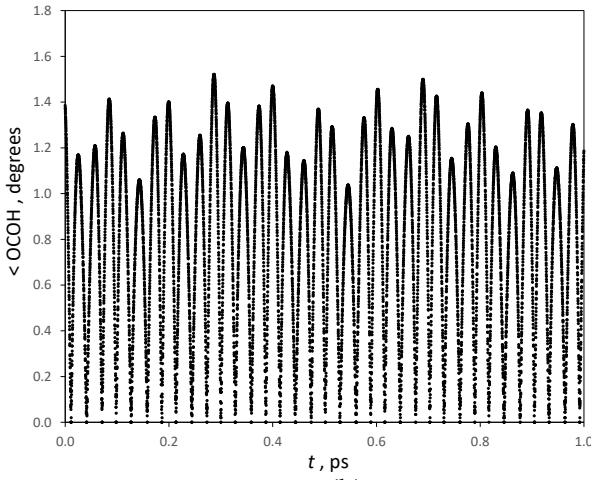

(b)

Fig. 7. The time-evolution of the $\mathrm{OCOH}$ dihedral angle throughout the first picosecond of the production ADMP runs at $5 \mathrm{~K}$, for the trajectories started from the cis-minima (a), and trans-minima (b) on the DFTB PES 


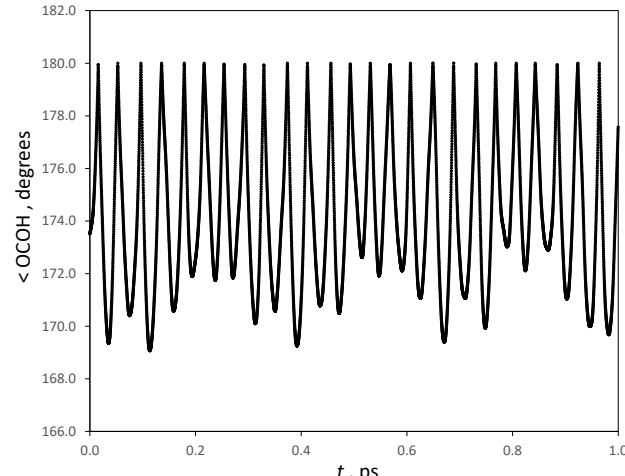

(a)

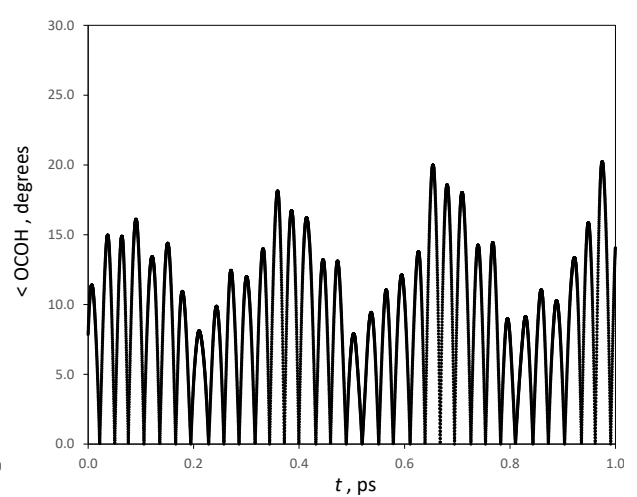

(b)

Fig. 8. The time-evolution of the $\mathrm{OCOH}$ dihedral angle throughout the first picosecond of the production ADMP runs at $300 \mathrm{~K}$, for the trajectories started from the cis-minima (a), and trans-minima (b) on the DFTB PES

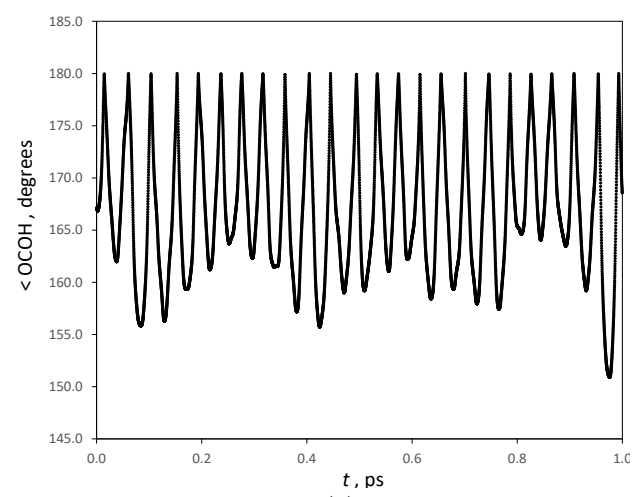

(a)

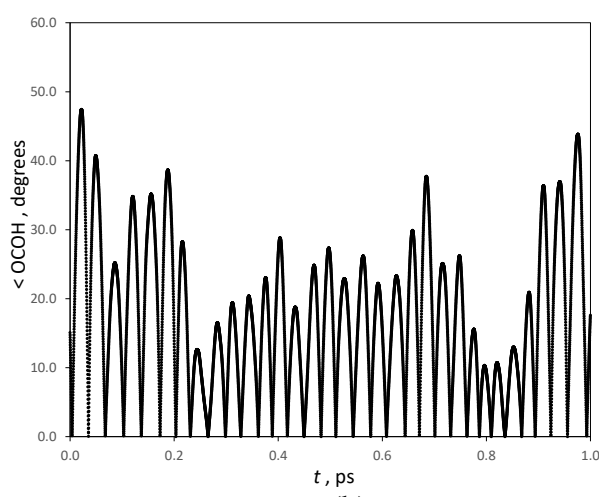

(b)

Fig. 9. The time-evolution of the $\mathrm{OCOH}$ dihedral angle throughout the first picosecond of the production ADMP runs at $1000 \mathrm{~K}$, for the trajectories started from the cis-minima (a), and trans-minima (b) on the DFTB PES

Table 1 . The average absolute deviations of the torsional angle values

\begin{tabular}{|c|c|c|}
\hline \multirow{2}{*}{$T, \mathrm{~K}$} & \multicolumn{2}{|c|}{$\left|D-D_{0}\right|,^{\circ}$} \\
\cline { 2 - 3 } & cis-minima & trans-minima \\
\hline 5 & 0.66 & 0.82 \\
\hline 300 & 5.80 & 11.32 \\
\hline 1000 & 11.13 & 15.38 \\
\hline
\end{tabular}

In Table 1 are shown the average absolute deviations of the torsional angle values (with respect to the referent ones, corresponding to the minima on the DFTB PES) computed from the complete production phases of DFTB-ADMP runs at $5 \mathrm{~K}$, $300 \mathrm{~K}$ and $1000 \mathrm{~K}$, for the trajectories started from the cis- and trans-minima on the DFTB PES. An interesting observation concerning the average absolute deviations presented in Table 1 concerns the fact that at each temperature, the values for the trans conformer are slightly larger than the corresponding ones for the cis-conformer. We attribute this to the fact that the rise of the torsional potential is steeper from the cis-side of the well than from the trans, i.e., 


$$
\left|\left(\frac{d V}{d D}\right)_{\text {cis }}\right|>\left|\left(\frac{d V}{d D}\right)_{\text {trans }}\right| .
$$

It is also worth noting that the O-H stretching vibrational frequency shift upon trans $\rightarrow$ cis torsional transition, as computed from the positions of the corresponding VDOS bands in the spectra from trajectories at $5 \mathrm{~K}$ is about $50 \mathrm{~cm}^{-1}$, as compared to the experimental data of $67.5 \mathrm{~cm}^{-1}$ [10]. Note that the former value has been obtained without any scaling factors. This estimation is far better than our previous estimates, based on MP2-BOMD data [10].

To get a deeper insight into the correlation between the torsional motions and the $\mathrm{O}-\mathrm{H}$ stretching vibration as a function of temperature, we have also computed the momentary values of the $\mathrm{OCOH}$ dihedral angle for all time points during the production phase of the ADMP runs. The corresponding distribution histograms at all temperatures, calculated for the trajectories started from both cis- and transminima on the DFTB PES are presented in Figs 10 and 11. The appearance of the "Density Of States" (DOS) histograms is in line with the previous conclusions, concerning the enhancement of the number of different local intramolecular environments that the $\mathrm{O}-\mathrm{H}$ stretching oscillator experiences in the course of temperature increase.

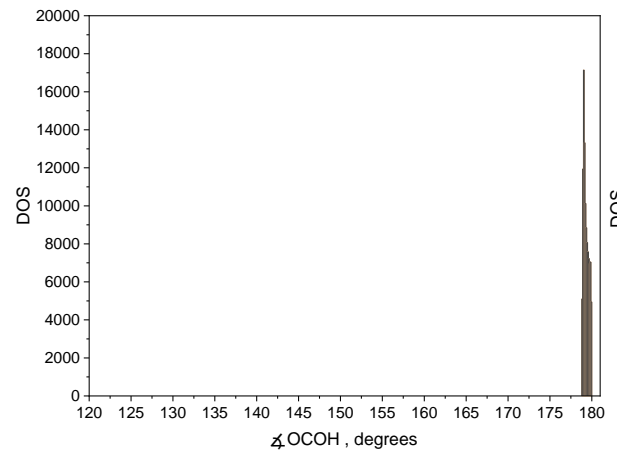

(a)

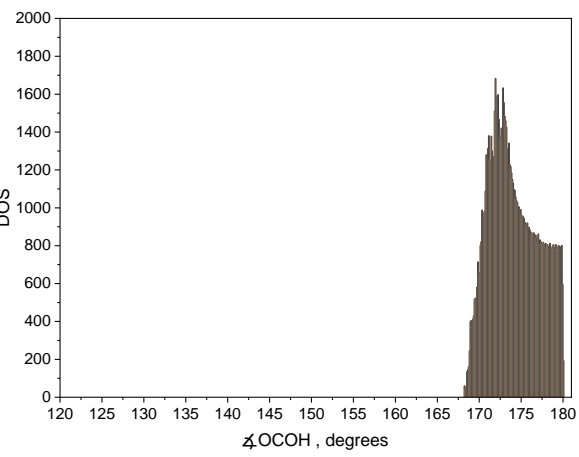

(b)

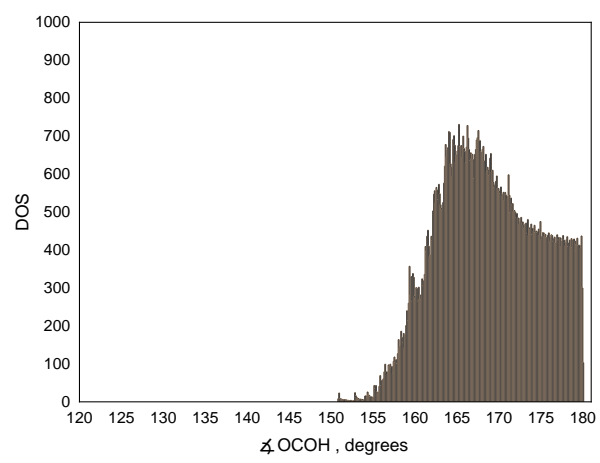

(c)

Fig. 10. Histograms of distribution of the $\mathrm{OCOH}$ dihedral angle values throughout the production ADMP runs at $5 \mathrm{~K}(\mathrm{a}), 300 \mathrm{~K}$ (b), and $1000 \mathrm{~K}$ (c) for the trajectories started from the cis-minimum on the DFTB PES 


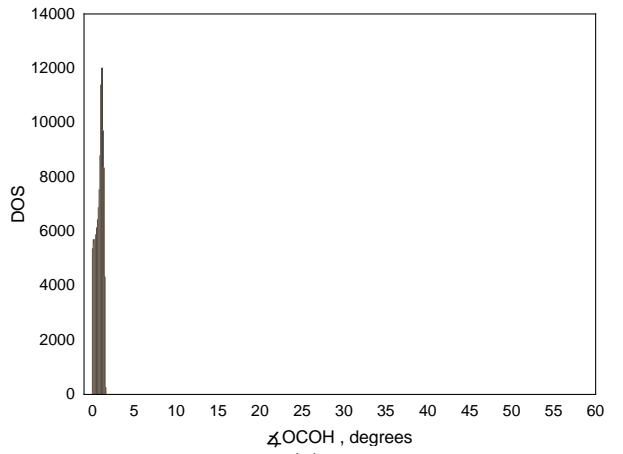

(a)

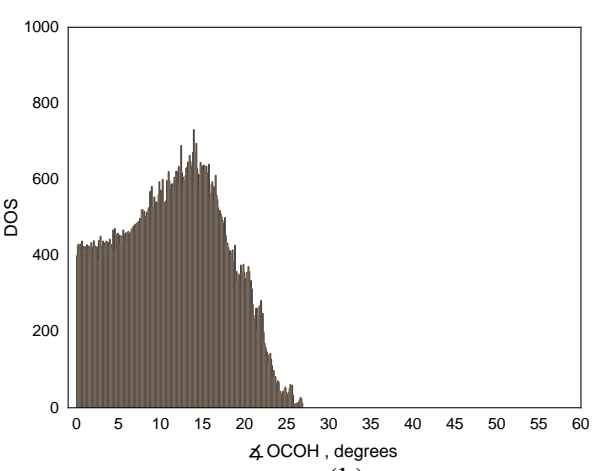

(b)

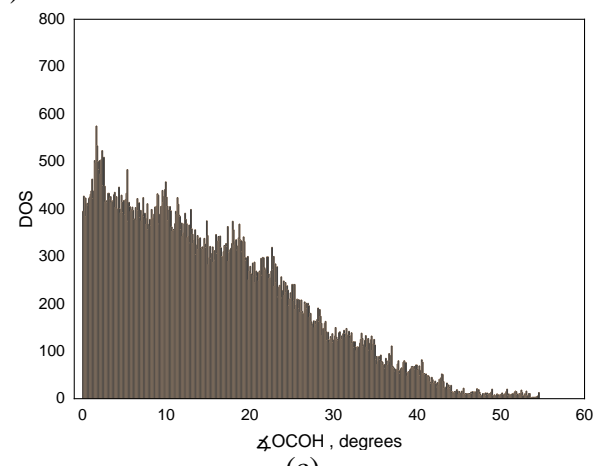

(c)

Fig. 11. Histograms of distribution of the $\mathrm{OCOH}$ dihedral angle values throughout the production ADMP runs at $5 \mathrm{~K}$ (a), $300 \mathrm{~K}$ (b) and $1000 \mathrm{~K}$ (c) for the trajectories started from the trans-minimum on the DFTB PES

\section{Conclusion}

To enable realistic comparisons with the experimentally obtained single-molecule vibrational spectroscopic data at finite temperatures (usually far above absolute zero), one cannot rely only on standard single-point molecular quantum mechanics calculations. We have demonstrated in the present study that the spectral appearances in the regions of $\mathrm{O}-\mathrm{H}$ stretching modes in the case of the simplest organic acid molecule (formic acid) are strongly temperature-dependent. Generally, temperature increase causes frequency downshift (red-shift) for a particular O-H oscillator. We attribute this observation to the fact that upon temperature increase, as a consequence of the increased intramolecular thermal motions, the $\mathrm{O}-\mathrm{H}$ oscillators sample more efficiently more distant (from the minimum) regions of the vibrational potentials, which is equivalent to they becoming "more anharmonic". At the same time, as a consequence of the enhanced $\mathrm{OCOH}$ torsional motion upon temperature increase, the $\mathrm{O}-\mathrm{H}$ oscillators sample much more different local intramolecular "environments", resulting in the corresponding spectral bands being significantly broadened and substructured. The present study opens a number of new issues concerning the development of theoretical methodologies aiming to provide a solid theoretical support to interpretation of experimental vibrational spectroscopic data. 


\section{References}

1. Domanskaya, A., K. Marushkevich, L. Khriachtchev, M. Rasanen. Spectroscopic Study of Cis-to-Trans Tunnelling Reaction of HCOOD in Rare Gas Matrices. The Journal of Chemical Physics, Vol. 130, 2009, 154509.

2. Koteska, B., A. Mishev, L. Pejov. Dynamic Versus Static Approach to Theoretical Anharmonic Vibrational Spectroscopy of Molecular Species Relevant to Atmospheric Chemistry: A Case Study of Formic Acid. - Scalable Computing: Practice and Experience, Vol. 19, 2018, 119.

3. Elst ner, M., D. P ore zag, G. Jungnickel, J. E1sner, M. Haugk, T. Frau en hei m, S. S u h a i, G. S e if e rt. Selfconsistent-Charge Density-Functional Tight-Binding Method for Simulations of Complex Materials Properties. - Physical Review B, Vol. 58, 1998, 7260.

4. Zheng, G., H. A. Witek, P. B obadova-Parvanova, S. Irle, D. G. Musaev, R. Prabhakar, K. Morokuma, M. Lundberg, M. Elstner, C. Kohler, T. Fraue nheim. Parameter Calibration of Transition-Metal Elements for the SpinPolarized Self-Consistent-Charge Density-Functional Tight-Binding (DFTB) Method: Sc, Ti, Fe, Co and Ti. - Journal of Chemical Theory and Computation, Vol. 3, 2007, 1349.

5. S chlegel, H. B., J. M. Milla m, S. S. Iyengar, G. A. Voth, A. D. D an ie ls, G. E. S c u s e ri a, M. J. Fri s c h. Ab Initio Molecular Dynamics: Propagating the Density Matrix with Gaussian Orbitals. - The Journal of Chemical Physics, Vol. 114, 2001, 9758.

6. Fr i s c h, M. J., et al. Gaussian 09. Revision A.01. Wallingford CT, Gaussian, Inc., 2009.

7. P e n g, C., P. Y. A y a l a, H. B. S c h l e g e l. Using Redundant Internal Coordinates to Optimize Equilibrium Geometries and Transition States. - Journal of Computational Chemistry, Vol. 17, 1996, 49.

8. Petters son, M., E. M. S. Macoas, L. Khriachtchev, R. Fausto, M. Ras anen. Conformational Isomerization of Formic Acid by Vibrational Excitation at Energies Below the Torsional Barrier. - Journal of the American Chemical Society, Vol. 125, 2003, 4058.

9. Li, X., J. Oomens, J. R. Eyler, D. T. Moore, S. S. Iy engar. Isotope Dependent, Temperature Regulated, Energy Repartitioning in a Low-Barrier, Short-Strong Hydrogen Bonded Cluster. - The Journal of Chemical Physics, Vol. 132, 2010, 244301.

10. K o t e s k a, B., A. M i s h e v, L. P e j o v. Computational Study of Intramolecular O-H Stretching Vibrations in the Two Rotamers of Free Formic Acid. - Romanian Reports in Physics, Vol. 70, 2018, 204.

Received: 25.08.2020; Second Version: 4.11.2020; Accepted: 5.11.2020 